\title{
The first sixty minutes in preterm neonates' life: predicted morbidities and interventions
}

\begin{abstract}
Preterm neonates are more vulnerable to many diseases and complications than full-term infants as most of their biological systems are still underdeveloped. Fortunately, most of these morbidities are preventable as there are many interventions that could be performed in the first 60 minutes of neonatal life to avoid them and their long-term consequences, thus rightfully defining 'the golden hour'. The objective of this article is to review the potential morbidities that neonates might experience and understand their mechanisms, risk factors and its possible outcomes. We also reviewed the possible actions that could be implemented during the golden hour to prevent these morbidities and investigated their effectiveness. Medline and Cochrane database for systematic review were the main two electronic database that we used to search for studies. Studies pertaining to morbidities, such as hypothermia, hypoglycemia, respiratory distress, intraventricular hemorrhage (IVH), Chronic lung disease (CLD) and retinopathy of prematurity (ROP) have been selected according to their full text accessibility for us. Reports related to possible interventions that could be performed to prevent these morbidities have also been involved that include but not only restricted to drying \& head coverings, polyethylene occlusive skin wraps, ambient room temperature, pre-warmed incubator, heated humidified gases, heated mattress and skin-to-skin contact for hypothermia; dextrose infusion pump, dextrose burette and oral dextrose for hypoglycemia; indomethacin, ibuprofen, antithrombin and heparin for IVH; surfactant and CPAP for respiratory distress; controlling oxygen saturation for ROP
\end{abstract}

Volume 8 Issue I - 2018

\author{
Khaled El-Atawi,' Mahmoud Elhalik, \\ Tushar Kulkarni, ${ }^{3}$ Ahmed Zakaria, ${ }^{4}$ Amany \\ Abdelsamed, ${ }^{4}$ Lois Alexander, ${ }^{4}$ Aswathy \\ Devaki Satyan ${ }^{4}$ \\ Consultant Neonatologist, Neonatal Intensive Care, Pediatric \\ Department, Latifa Hospital, Dubai Health Authority, UAE \\ ${ }^{2}$ Consultant Neonatologist and Head of Pediatric Department \\ Neonatal Intensive Care, Pediatric Department, Latifa Hospital, \\ Dubai Health Authority, UAE \\ ${ }^{3}$ Specialist Senior Registrar, Neonatal Intensive Care, Pediatric \\ Department, Latifa Hospital, Dubai Health Authority, UAE \\ ${ }^{4}$ Specialist Registrar, Neonatal Intensive Care, Pediatric \\ Department, Latifa Hospital, Dubai Health Authority, UAE
}

Correspondence: Khaled El-Atawi, Consultant Neonatologist, Neonatal Intensive Care, Pediatric Department, Latifa Hospital, Dubai Health Authority, UAE, Email kelatawi@eim.ae

Received: December 10, 2017| Published: February 12, 2018

Keywords: golden hour, preterm, hypothermia, hypoglycemia, retinopathy of prematurity, respiratory distress, intraventricular hemorrhage, chronic lung disease

Abbreviations: IVH, intraventricular hemorrhage; CLD, chronic lung disease; ROP, retinopathy of prematurity

\section{Introduction}

The sudden movement of preterm neonates from the warm environment of the womb to the cold one of delivery rooms along with the immature protective mechanisms and biological systems of newborns make them fragile and susceptible to a variety of morbidities. Creating a delivery room with a complete thermo-neutral medium (a medium in which oxygen consumption is minimal) is an unattainable target which makes external interventions to achieve infants' wellbeing an inevitable issue. During the initial sixty minutes of neonatal life "the golden hour", how health care providers intervene to prevent these morbidities could affect their short \& long-term consequences that range from mild defects up to permanent hearing and vision loss and death. ${ }^{1}$ The term "the golden hour" has primarily been introduced to scientific literature by traumatology disciplines referring to the first hour of treatment of traumatized patients. ${ }^{2}$ In this review, we have covered multiple aspects of different complications including hypothermia, hypoglycemia, respiratory distress, intraventricular hemorrhage (IVH) and retinopathy of prematurity (ROP). Each one of these morbidities has been discussed separately in terms of its mechanism of detriment, short and long-term complications, potential techniques and medications that could be provided to bypass these complications.

\section{Hypothermia}

Hypothermia is a worldwide problem that has heavy impacts and consequences on preterm neonates. The World Health organization (WHO) has laid down the classification based on the severity of hypothermia as mild $\left(36.0-36.4^{\circ} \mathrm{C}\right)$; moderate $\left(32-35.9^{\circ} \mathrm{C}\right)$; severe $\left(<32^{\circ} \mathrm{C}\right) .^{3}$ Neonatal hypothermia is highly prevalent all over the world, it is estimated to be $32 \%-85 \%$ inside hospitals. The fatality rate is estimated to be $39.3 \%$ in mildly hypothermic babies, $51.6 \%$ in moderately hypothermic babies and $80 \%$ in severely hypothermic babies. ${ }^{4}$ Birth weight acts as the key element in predicting hypothermia at admission in very low birth weight (VLBW). ${ }^{5,6}$ Mean rectal temperature on admission to neonatal intensive care unit (NICU) increases by $0.21^{\circ} \mathrm{C}(95 \% \mathrm{CI}, 0.04$ to 0.4$)$ with each 100 gm increase in birth weight.?

Hypothermia is found to be associated with morbidity especially if it's prolonged. It can be associated with hypoglycemia, acidosis, late onset sepsis and chronic lung disease. Moderate hypothermia $\left(32-35.9^{\circ} \mathrm{C}\right)$ is associated with respiratory distress syndrome and mortality but it doesn't correlate with neurodevelopmental delay. ${ }^{5}$ Additional studies to illustrate the relation between severe hypothermia $\left(<32^{\circ} \mathrm{C}\right)$ and short-and long-term neurodevelopmental outcomes are required. Moderate hypothermia was found to be associated with severe intraventricular hemorrhage, intraventricular leukomalacia, and necrotizing enterocolitis. Yet, these associations should be analyzed carefully since they were based on studies with a wide confidence interval. More studies with larger sample size are warranted to accurately illustrate these associations. ${ }^{5}$ Moreover, hypothermia increases oxygen consumption, thus leading to hypoxia and subsequently pulmonary vasoconstriction which, in turn, declines surfactant production and worsens respiratory distress. The presence of comorbidities with hypothermia increases the fatality rate, so adding a higher category to the severity of hypothermia would represent an effective addition to the WHO classification of the severity of hypothermia. ${ }^{8}$

Large surface area of neonates in relation to their small body weight makes them more vulnerable to hypothermia than full-term 
babies. Preterm neonates have a higher transepidermal water loss than term due to their immature epidermal layer. ${ }^{9}$ Their premature vasomotor control which acts as a thermoregulatory mechanism has an impact in the causal pathway of hypothermia incidence in neonates. ${ }^{10}$ Furthermore, neonates have a little subcutaneous fat store which is supposed to act as an insulating layer against heat loss and plays a role in thermogenesis. Moreover, environmental factors in delivery rooms also contribute to hypothermia. When newborns move from the uterine medium to the cool environment of delivery rooms, amniotic fluid that evaporates from newborns' skin due to the low temperature and humidity of the ambient air represents one of the main mechanisms of heat loss. Additionally, convection heat loss which occurs secondary to the circulation of cooler air across neonates' skin and mucous membrane, conductive heat loss as babies contacting with cooler bed sheets. Eventually, the low temperature of the surrounding materials in delivery rooms leads to radiation heat loss. All these factors substantially contribute to hypothermia in preterm.

Healthcare providers should intervene instantly after birth in order to prevent hypothermia as heat production secondary to fat degradation can't compensate the heat loss. Hypothermia prevention before neonatal transportation leads to a better outcome. ${ }^{11-13}$ The distance during neonatal transportation is considered an insignificant factor in comparison with hypothermia stabilization. ${ }^{11}$ A variety of interventions and well-trained dedicated care providers are required to optimize the body temperature of preterm neonates as the process of heat loss are caused by multiple factors. External heat sources are usually required to maintain those high-risk population at the normothermic range.

\section{What interventions could be implemented to prevent heat loss?}

Drying \& head coverings: Drying, swaddling in a blanket and positioning under overhead heaters have long been the cornerstone of thermal care procedures for neonates in delivery rooms. Since the head constitutes a major portion of the body surface area in neonates and the brain plays the role of a prime heat-generating organ, head covering has been an effective technique in preventing temperature reduction in neonates. ${ }^{14}$ But all these techniques are still insufficient in very preterm neonates.

Ambient temperature of delivery room: According to WHO's recommendation the temperature of delivery rooms' should be adjusted at no less than $25^{\circ} \mathrm{C}$, while the International Liaison Committee on Resuscitation (ILCOR) 2010 recommends that it should be $\geq 26{ }^{\circ} \mathrm{C} . .^{3-15}$ However, these suggestions aren't feasible targets. Delivery rooms' temperature is often adjusted according to health care providers' preferences regardless neonates' needs. Furthermore, there are a number of delivery rooms that don't have a separate thermostat, and pre-set temperatures resulting in room temperature variation. ${ }^{16}$ Additionally, the temperature and humidity of delivery room should be measured soon after birth and newborn's temperature should be documented every ten to fifteen minutes until the developing of a system able to observe temperature continuously. ${ }^{17}$ The 7 th edition of the Neonatal Resuscitation Program (NRP) suggests that the temperature in the delivery room should be increased to $23{ }^{\circ} \mathrm{C}-25^{\circ} \mathrm{C}$.

Polyethylene occlusive wrap: There are a large number of proofs or past incidents that swaddling preterm neonates with plastic bag immediately after birth reduces heat loss and is as effective as standard thermoregulation procedures. ${ }^{18-20}$ Yet, it doesn't reduce heat loss in neonates aged 28 to 31 weeks. ${ }^{21}$ Plastic wrap prevents thermal loss through increasing the humidity between the skin and the sheath which diminishes evaporative heat loss. It also acquires heat from radiant warmers by radiation..$^{22}$ Past study findings have stated that polyethylene occlusive skin wrapping prevent heat loss in very preterm neonates. ${ }^{7}$ With regard to whether to cover the whole body or exclude the head, a recent report found that total body wrapping can be equalized to covering the body till the shoulders in preventing heat loss in very preterm infants. ${ }^{23}$

Although, almost all studies found that plastic wrap is related to a reduction in hypothermia in preterm neonates, these studies didn't find a substantial decrease morbidity and mortality when using plastic wraps. ${ }^{24}$ Additionally, evidence that suggests plastic wrap coverings reduce the risk of intraventricular hemorrhage is statistically insignificant. ${ }^{20,25,26}$ Moreover, past findings reveal that plastic wraps do not completely decrease the risk of respiratory distress syndrome. ${ }^{20}$ Further studies to investigate the impact of plastic wraps on neonatal morbidity and mortality are warranted.

Plastic wraps combined with external heat sources have been found to be effective in preterm neonates in reducing hypothermia when transporting inside hospitals. The effect of plastic wrap coverings outside hospital setting or without additional heat source are not well documented \& more studies to illustrate this issue are required. In terms of hyperthermia that could occur as an adverse effect with the use of plastic wraps, there are conflicting reports in this regard. Four randomized controlled trials concluded that hyperthermia isn't associated with plastic covering, while other studies that investigated the effect of plastic wrap among other procedures as a thermoregulatory bundle in VLBW indicated that there is no increase in hyperthermia rates with the use of polyethylene wraps. Similarly, clinical trial that has been conducted on 96 neonates aged 29-32 weeks found that there is no risk of hyperthermia with plastic bags. ${ }^{19-29}$

Heated mattress: Conductive thermal mattress (CTM) has been found to be as efficacious as radiant warmer and other modes of warming that are often used as standards of thermal care. This was reported with regard to its short term use,$^{30}$ however further trials are required to assess the long-term effects.

A report that used a standardized multidisciplinary approach in which a chemically activated heated mattress is combined with other techniques, such as polyethylene wraps, servo-controlled radiant warmer, warming light, newborn hats, and a well-trained dedicated team to monitor the axillary temperature every 5 minutes achieved success in preventing hypothermia in very low-birth-weight babies whose weight is below $1500 \mathrm{gm}^{31}$. Hyperthermia was reported as an adverse effect in this protocol, and it was addressed by decreasing the temperature of beds during resuscitation from $37.5^{\circ} \mathrm{C}$ to $37^{\circ} \mathrm{C}$ and removing the polyethylene wrap after the admission temperature amounted to $36.5^{\circ} \mathrm{C}$. However, studies to examine the effect of the thermal mattress on extremely low-birth-weight whose weight is less than $1000 \mathrm{gm}$ are needed since it hasn't been investigated yet.

Skin-to-skin contact (SSC): Very early SSC has long been used as an effective technique to prevent heat loss and to maintain a normal body temperature in term and late preterm vaginally-delivered newborn. ${ }^{32,33}$ This has been achieved by putting a nude baby on mother's unclad chest within 30 or 40 minutes after birth. ${ }^{34}$ The use of SSC after cesarean section was an unusual practice due to the risk of hypothermia. yet recent randomized clinical trials that examined the effect of SSC after cesarean section concluded that SSC doesn't increase the risk of hypothermia. ${ }^{35,36}$

Neonates accompanied by health care professionals such as doctors and nurses have a poorer outcome than those who are accompanied 
by their parents during transportation from delivery room to NICU. ${ }^{11}$ Additionally, A randomized controlled trial that compares between the effect of SSC and conventional incubator on physiological stabilization revealed the following stabilization scores: 77.11 for ssc versus 74.23 for incubator, mean difference 2.88 (95\% CI: 0.3-5.46, $\mathrm{p}=0.031$ ). Skin-to-skin contact between the mothers and their babies gives rise to improved physiological results and stability as compared to the similar care provided in incubators, thus newborn shouldn't be separated from their mothers. ${ }^{37}$

Pre-warmed incubator: Double walled pre-warmed incubator has long been used to preserve body temperature in preterm newborn. It provides a medium that mimic the intrauterine environment, hence preventing evaporative and convective heat loss. However, infants aren't easily accessible when they are in the incubator, therefore it's not that practical tool for infants who need multiple interventions for other morbidities. Moreover, it isn't a handy device in resource-limited settings such as developing countries. The warm moist environment of the incubator represents an ideal media for bacteria to proliferate which increases the risk of staphylococcal infection. ${ }^{38} \mathrm{~A}$ recent systematic review concluded that there is no risk of hyperthermia with synchronized use of incubators $(5.4 \%$ with incubator use, $8.1 \%$ without, $\mathrm{RR}=0.66 ; 95 \% \mathrm{CI} 0.19$ to 2.3$).^{24}$

Heated humidified gases (HHG): Historically, heated humidified gases has been used for respiratory support in neonates who are suffering from respiratory distress. Recently, it has been introduced as a tool to preserve body temperature for very preterm infants. A statistically significant difference was noted in rectal temperature between the cold and heated cohorts $\left(35.9^{0.6}\right.$. vs $36.4^{0.6}$., respectively; $\mathrm{P}<.0001) .{ }^{39} \mathrm{~A}$ randomized controlled trial that compared between HHG and cold dry gas concluded that in the HHG group, $69 \%$ of preterm neonates were normothermic compared with $55 \%$ in the cold, dry gas group (unadjusted OR 1.8, 95\% CI 1.01-3.19). ${ }^{40}$

\section{Hypoglycemia}

Hypoglycemia is one of the most common major problems that contributes to neonatal morbidity and mortality. There is no scientifically documented definition for neonatal hypoglycemia; however, plasma blood glucose lower than $47 \mathrm{mg} / \mathrm{dl}$ is widely acknowledged as a cut-off value below which newborns are considered hypoglycemic. ${ }^{41}$ Infants born to diabetic mothers, large for gestational age (LGA), small for gestational age (SGA), low birth weight and late preterm are at a higher risk of developing hypoglycemia. ${ }^{41-43}$ Another study found that a pre-pregnancy BMI greater than $25 \mathrm{~kg} / \mathrm{m} 2$ in women with GDM also predicts neonatal hypoglycemia independent of maternal glucose concentrations. ${ }^{44}$ Furthermore, male sex is found to be associated with neonatal hypoglycemia. ${ }^{45}$

Neonatal hypoglycemia is considered a risk factor for brain pathology and neurodevelopmental impairment in moderately preterm and term infants. ${ }^{46,47}$ The frequency, rather than the severity of hypoglycemic episodes, predicts the adverse long-term effects. Therefore recurrent monitoring of blood glucose in high-risk groups is recommended. ${ }^{47}$ However, there is no particular cut off value of blood glucose concentration or hypoglycemic episode duration at which one can expect long-term complications. Despite almost all neonates that experience a transient asymptomatic low blood glucose up to 30 $\mathrm{mg} / \mathrm{dl}$ in the first few hours of their lives as a normal adaptation to postnatal life, a recent study indicated that there is a relation between transient neonatal hypoglycemia (in the first three hours of life) and poor academic achievement at 10 years old of age. ${ }^{48}$ Further studies in this regard are warranted to assess the generalizability of this conclusion - which represents an essential step before endorsing a universal newborn blood glucose screening program. A study that examined tissue glucose (TG) concentrations in VLBW infants even after stabilization in NICU found that fluctuations of TG may vary from $2.5 \mathrm{mg} / \mathrm{dl}$ to $8.3 \mathrm{mg} / \mathrm{dl}$ which makes these high-risk group more vulnerable to metabolic syndrome. ${ }^{49}$

Currently, the typical treatment of neonatal hypoglycemia is IV fluid administration which necessitates infant-mother separation, thus impedes SSC and hinders breastfeeding. In an attempt to handle these adverse effects, a group of researchers implemented a new protocol to address neonatal hypoglycemia. They found that oral administration of $40 \%$ glucose gel declines the rate of admission to NICU for hypoglycemia by $73 \%$ and subsequently bypass the abovementioned adverse effects of IV fluid therapy. ${ }^{50}$ Another study that involved a maximum of three doses of dextrose gel $(200 \mathrm{mg} / \mathrm{kg}$ of $40 \%$ dextrose) in addition to the feeds of asymptomatic hypoglycemic neonates showed a similar conclusion. ${ }^{51}$ A randomized controlled trial was carried out in an effort to optimize the dose of oral dextrose and showed that the incidence of neonatal hypoglycemia can be lessened with a single dose of buccal $40 \%$ dextrose gel $200 \mathrm{mg} / \mathrm{kg} .{ }^{52}$

Management of neonatal hypoglycemia has long been controversial and poorly defined, let alone how challenging it's in resources-limited settings. Since dextrose infusion pump is not usually handy in poor countries, a group of researchers in Uganda investigated the effect of dextrose infusion though burette, among preterm, as an alternative to dextrose pump concluded that continuous $10 \%$ dextrose infusion by burettes reduces the incidence of hypoglycemia by $81 \%$ compared to dextrose boluses. ${ }^{53}$

A recent clinical report recommended that blood glucose concentration should only be measured in the first minutes of life of term infants for the ones who have had clinical manifestations or are exposed to higher levels of risk, including SGA, LGA, born to a diabetic mother and late pre-term. ${ }^{41}$

Episodes of hypoglycemia are often subtle and clinically silent and whether to address these asymptomatic episodes in preterm remains an unsettled issue. However, hypoglycemia due to hyperinsulinism are recommended to immediately undergo early diagnosis and treatment because they more prone to brain injury. ${ }^{54,55}$

Early administration of parenteral nutrition immediately after birth aims at minimizing postnatal deprivation of amino acids, glucose and nutrients as the prematurely born neonate is adapting to extrauterine life. Early initiation of parenteral amino acids is also known to stimulate insulin secretion in the premature infant in the absence of which insulin dependent pathways of transporter mediated glucose transfer maybe affected leading to disruption of $\mathrm{Na}$, K ATPase activity and resultant intracellular energy failure and hyperkalemia. ${ }^{56,57}$

Intraventricular hemorrhage (IVH): About 15 - 20 percent of preterm infants experience IVH and its incidence has slightly been increased over the last decade. ${ }^{54}$ Ten to fifteen percent of neonates $<$ 1500 gm birth weight experience severe grades of hemorrhage..$^{56}$ There are several risk factors for IVH, such as LGA, lack or non-presence of antenatal steroid exposure, antenatal maternal hemorrhage, neonatal transport, maternal chorioamnionitis/ infection/ inflammation, maternal fertility treatment, early sepsis, hypotension, therapeutic intervention, pulmonary hemorrhage, hypoxemia, hypercapnia, respiratory distress syndrome (RDS), pneumothorax, severity of illness score, convulsion, SGA status, acidosis treatment, and pressor treatment. ${ }^{56}$

Nearly three-quarter of infants with grade 3 or 4 IVH would experience complications, such as cerebral palsy, post-hemorrhagic 
ventricular dilatation and intellectual disabilities. ${ }^{54}$ There is no obtainable therapy to prevent these consequences. Neonates with high grades of IVH may experience death secondary to hypovolemic shock and hydrocephalus. ${ }^{56}$

IVH occurs more commonly in the first 24 hours of preterms life, so preventive agents should be administered before this risk period. Indomethacin and Ibuprofen have been found to be equally effective in reducing the incidence of IVH. ${ }^{56,58} \mathrm{~A}$ recent review found that antithrombin administration doesn't reduce the incidence of IVH in very low-birth-weight preterm when compared to placebo, no treatment, and heparin. ${ }^{59}$ Additional studies are warranted to illustrate the effect of heparin as a preventive agent for IVH. ${ }^{60}$

Lung Injury: Surfactant deficiency, immature lung structure, and poorly developed respiratory mechanisms are the main causes of lung injury among preterm neonates. Therefore, respiratory support is generally recommended for that high-risk group to maintain functional residual capacity and avoid lung damage..$^{56,61}$

Surfactant administration at birth of preterm infants is considered a favorable option as part of respiratory support. Giving surfactant prophylactically at birth to high-risk infants is found to reduce mortality and air leak than selectively administering it to an infant with established RDS. ${ }^{62}$ In contrast early stabilization on CPAP with subsequent selective administration of surfactant to extremely preterm infants reduces the risk of bronchopulmonary dysplasia and death compared with early administration of surfactant. Furthermore, the risk of adverse effects does not increase if neonates are stabilized on CPAP alone without surfactant. Early CPAP followed by early administration of selective surfactant is regarded as an effective substitute to routine intubation and prophylactic surfactant. ${ }^{62}$

The majority of resuscitation guidelines recommend CPAP for respiratory support for preterm neonates, CPAP declines the duration of mechanical ventilation. PEEP has been shown to facilitate gas exchange, improve lung mechanics in preterm lamb, so CPAP should not be used in place of positive pressure ventilation when respiratory effort is poor or absent. ${ }^{15-64}$

Sustained inflation has been developed as an alternative to CPAP. The European resuscitation council recommended that sustained inflation should be used for preterm infants who suffer from apnea as it has been found to improve lung ventilation. The duration and pressure at which it should be applied aren't optimized yet. Sustained inflation extends the inspiratory phase which subsequently prompts more alveoli to expand and enhance pulmonary blood flow. Furthermore, it facilitates lung expansion and improves the compliance. ${ }^{15-63}$

Chronic lung disease (CLD), also known as bronchopulmonary dysplasia, is one of the most common complications of premature birth. Newborn babies suffer from CLD due to the lung injuries in newborns kept on mechanical ventilator requiring extra oxygen for breathing. It can be diagnosed in case a newborn is experiencing respiratory distress like rapid breathing, flaring of the nostrils, grunting, and chest retractions or requirement of mechanical ventilator even after reaching 36 weeks of gestation. Although all the causes of premature births cannot be prevented, a healthy pregnancy can certainly result in decreasing the chances of infants having immature lungs while their birth. ${ }^{59,65}$

A systematic review and metanalysis of Georg et al. showed that as compared to IPPV, preterm infants initially treated with SI at birth required less mechanical ventilation with no improvement in the rate of BPD and/or death. The use of SI should be restricted to randomized trials until future studies demonstrate the efficacy and safety of this lung aeration manoeuvre. ${ }^{59,66}$
The ideal fraction of inspired oxygen $\left(\mathrm{O}_{2}\right)$ has not been optimized yet. A recent report found that there is no difference in the risk of death or other morbidities after resuscitation with either lower $(\leq 0.30)$ or higher $(\geq 0.6) \mathrm{FiO}_{2}$ in infants $\leq 28+6$ weeks gestation. ${ }^{67}$ Each hospital must set an optimal target according to their conditions and all health care providers should be informed of these values and supplemental oxygen should be adjusted accordingly ${ }^{68}$ Another study that investigated the effect of $100 \%$ oxygen or room air (RA) on $\leq 28$ weeks infants found a significantly higher hospital mortality among room air group: $22 \%$; than those given $100 \% \mathrm{O} 2$ : $6 \%$; risk ratio: 3.9 $95 \%$ confidence interval: $1.1-13.4$.; $\mathrm{P}=.01) .{ }^{69}$

A group of researchers recently investigated the effect of Caffeine on 59 preterm infants with respiratory distress syndrome (RDS). They found that Caffeine significantly reduces the intubation time, the total duration of oxygen supply, time of NCPAP and the rate of ventilatorassociated pneumonia. ${ }^{70}$

Retinopathy of Prematurity (ROP): Nearly 32,200 infants become blind every year due to retinopathy of prematurity ${ }^{71}$. Preterm neonate whose birth weight is $<1250 \mathrm{gm}$ or $<28$ weeks gestation are more susceptible to abnormal growth of blood vessels in their undervascularized retina which leads to what's known ROP. Monitoring oxygen therapy and avoiding intermittent hypoxia have been shown as the cornerstone factors in preventing ROP. Refractory errors, retinal detachment and recurrent ROP are the most common complications of $\mathrm{ROP}^{\text {.73-75 }}$

Retinopathy of Prematurity, to a great extent, is a preventable disease, so introducing strict qualified standards of neonatal care, such as restricted supervised oxygen use for preterm and screening program for early detection and treatment of ROP would be effective in reducing its incidence thus its consequences.

There are many medications that have been reported to induce ROP. The duration of steroid use increases the incidence of ROP, therefore the use of postnatal Dexamethasone has been restricted. Additionally, Indomethacin increases the risk of ROP by 1.5 times. There are conflicting reports with regards to the association between ROP and the use of surfactant ${ }^{73}$. Additional studies are needed to illustrate this relationship.

Applying different grades of targeted oxygen saturation according to gestational ages (83-89\% until 32week, 90-94\% until 35week and $>94 \%$ at $\geq 36$ week postmenstrual age) have been found to reduce the incidence of ROP (OR: $0.18,95 \%$ CI: $0.11,0.30$; P $<0.001$ ) and hasn't increased the mortality (OR: $0.74,95 \%$ CI: $0.37,1.49$; P $=0.40) \cdot{ }^{71}$ However, a meta-analysis concluded that despite the fact that low oxygen concentration has been detected to decline the risk of ROP, it actually increases mortality (RR $1.15,95 \%$ CI, 1.04-1.29). ${ }^{76}$

There is a growing body of evidence that no single therapy has proven to be effective and safe in preventing and treating ROP. ${ }^{73,76}$ Managing ROP effectively necessitates coordination between physicians from different disciplines, such as neonatologists, obstetricians, and ophthalmologists. This teamwork may be the cornerstone of success in early detection and treatment of ROP. All healthcare providers concerned with the care of preterm neonates should be informed of the optimal oxygen saturation and ROP screening guidelines. Parents should be cautious about the potential consequences of ROP. ${ }^{77}$

The American Academy of Pediatrics 2013 guidelines recommended ROP screening of infants at 24 to 27 weeks' gestational age. ${ }^{78}$ Kennedy and his team suggest that current screening guidelines should be changed to accommodate infants of 22 to 23 weeks. ${ }^{79}$ Future population-based studies are required to address this issue. 
Infection and Antibiotic Treatment: Premature babies generally have weak immune system as they get less time to build up their own antibodies in order to fight infection. Most of the antibodies usually get transferred from their mothers to the baby during the last few months of pregnancy. Intravenous catheters are often used in premature babies to administer drugs and medicines, which later becomes a passage for bacteria. As a result, premature babies are frequently being given antibiotics to battle against infection causing bacteria and other common germs found in newborns. ${ }^{80}$

Conclusion: The first 60 minutes of preterm neonates' life are the most crucial time for their entire life. There are many interventions that could be taken during this hour to prevent short \& long-term complications of preterm morbidity. Regarding hypothermia management, welltrained dedicated health care professionals are required to adjust the body temperature of preterm since the process of heat loss is multifactorial. There are many ways to prevent hypothermia during the transportation from the delivery room to neonatal intensive care unit; radiant warmer, incubators, skin-to skin contact, plastic wraps and thermal mattress. ${ }^{11-81}$ Polyethylene wraps are as efficacious as standard care in preventing hypothermia. Further studies to illustrate the effect of polyethylene wraps on neonatal morbidity and mortality are needed. The effect of polyethylene wraps outside hospitals setting and on neonates aged 28-32 weeks require further investigations. The long-term impacts of thermal mattress and its effect on extremely low-birth-weight (whose weight is less than $1000 \mathrm{gm}$ ) have not been examined yet. There is no risk of hyperthermia with pre-warmed incubator, but infection has frequently been reported as an adverse effect. Additional research to compare between the effects of radiant warmer and incubators with regard to their outcomes on extremely low birth weight infants is required..$^{82,83}$

Additional studies to illustrate the relation between severe hypothermia $\left(<32^{\circ} \mathrm{C}\right)$ and short-and long-term neurodevelopmental outcomes are required. More studies with larger sample size are warranted to accurately illustrate the association between moderate hypothermia and intraventricular hemorrhage, intraventricular leukomalacia and necrotizing enterocolitis.

Neonatal hypoglycemia is associated with poor neurological outcomes and it has been found that transient hypoglycemia affects the academic performance at 10 years of age. Managing episodes of asymptomatic hypoglycemic is still a debatable issue, but hypoglycemia due to insulin secretion problems should be addressed promptly.

In terms of IVH, additional studies to investigate the role of heparin as a prophylactic agent are required. Indomethacin and Ibuprofen are equally effective. Anti-thrombin has no value in preventing IVH.

Giving surfactant prophylactically at birth to high-risk infants is found to reduce mortality, however early CPAP followed by selective surfactant administration is considered an effective alternative option to prophylactic surfactant. Surprisingly, the fraction of inspired oxygen has no effect on the risk of death. Caffeine significantly reduces the intubation time, the total duration of oxygen supply.

Applying different grades of oxygen saturation according to gestational age has been found to reduce the incidence of ROP. Indomethacin increases the risk of ROP by 1.5 times. Studies with larger sample size are needed to accurately investigate the relation between surfactant use and ROP. There is no one therapy has been proven to be effective and safe in preventing and treating ROP. Screening guidelines for ROP should be changed to include infants of 22 to 23 weeks.

\section{Conflicts of interest}

There is no conflict of interest.

\section{Acknowledgments}

None.

\section{Funding}

None.

\section{References}

1. Lambeth TM, Rojas MA, Holmes AP, et al. First Golden Hour of Life: A Quality Improvement Initiative. Adv Neonatal Care. 2016;16(4):264-272.

2. Sasada M, Williamson K, Gabbott D. The golden hour and pre-hospital trauma care. Injury. 1995; 26(3):215-216.

3. http://apps.who.int/iris/bitstream/10665/63986/1/WHO_RHT_ MSM 97.2.pdf

4. Lunze K, Bloom DE, Jamison DT, et al. The global burden of neonatal hypothermia: systematic review of a major challenge for newborn survival. BMC Med. 2013;11:24.

5. Chang HY, Sung YH, Wang SM, et al. Short- and Long-Term Outcomes in Very Low Birth Weight Infants with Admission Hypothermia. PLoS One. 2015;10(7):e0131976.

6. Bartels DB, Kreienbrock L, Dammann O, et al. Population based study on the outcome of small for gestational age newborns. Arch Dis Child Fetal Neonatal Ed. 2005;90(1):F53-F59.

7. Vohra S, Roberts RS, Zhang B, et al. Heat Loss Prevention (HeLP) in the delivery room: A randomized controlled trial of polyethylene occlusive skin wrapping in very preterm infants. $J$ Pediatr. 2004;145(6):750-753.

8. Mathur NB, Krishnamurthy S, Mishra TK. Evaluation of WHO classification of hypothermia in sick extramural neonates as predictor of fatality. J Trop Pediatr. 2005;51(6):341-345.

9. Hammarlund K, Sedin G. Transepidermal water loss in newborn infants. VI. Heat exchange with the environment in relation to gestational age. Acta Paediatr Scand. 1982;71(2):191-196.

10. Knobel RB, Vohra S, Lehmann CU. Heat loss prevention in the delivery room for preterm infants: a national survey of newborn intensive care units. J Perinatol. 2005;25(8):514-518.

11. Rathod D, Adhisivam B, Bhat BV. Transport of sick neonates to a tertiary care hospital, South India: condition at arrival and outcome. Trop Doct. 2015;45(2):96-99

12. Hoque, Haaq $\mathrm{M}$, Islam $\mathrm{S}$, et al. Causes of neonatal admissions and deaths at a rural hospital in KwaZulu-Natal, South Africa. Southern African Journal of Infectious Diseases. 2010;26(1):26-29.

13. Okechukwu AA, Achonwa A. Morbidity and mortality patterns of admissions into the Special Care Baby Unit of University of Abuja Teaching Hospital, Gwagwalada, Nigeria. Niger $J$ Clin Pract. 2009;12(4):389-394.

14. Stratton D. Aural temperature of the newborn infant. Arch Dis Child. 1977;52(11):865-869.

15. Richmond S, Wyllie J. European Resuscitation Council Guidelines for Resuscitation 2010 Section 7. Resuscitation of babies at birth Resuscitation. 2010;81(10):1389-1399.

16. Vohra S, Reilly M, Rac VE, et al. Study protocol for multicentre randomized controlled trial of HeLP (Heat Loss Prevention) in the delivery room. Contemp Clin Trials. 2013;36(1):54-60. 
17. Bhatt DR, White R, Martin G, et al. Transitional hypothermia in preterm newborns. J Perinatol. 2000;7 27(Suppl 2):S45-S47.

18. Leadford AE, Warren JB, Manasyan A, et al. Plastic bags for prevention of hypothermia in preterm and low birth weight infants. Pediatrics.2013;132(1):e128-e134.

19. Talakoub S, Shahbazifard Z, Armanian AM, et al. Effect of two polyethylene covers in prevention of hypothermia among premature. Iran J Nurs Midwifery Res. 2015;20(3):322-326.

20. Rohana J, Khairina W, Boo NY, et al.Reducing hypothermia in preterm infants with polyethylene wrap. Pediatr Int. 2011;53(4):468-474.

21. Sophia Tsakiri, Kathleen Kennedy. Interventions to prevent hypothermia at birth in preterm and/or low birthweight babies. Evidence-Based Child Health: A Cochrane Review Journal. 2000; 1(1):328-330.

22. Laptook AR, Watkinson M. Temperature management in the delivery room. Semin Fetal Neonatal Med. 2008;13(6):383-391.

23. Doglioni N, Cavallin F, Mardegan V, et al. Total body polyethylene wraps for preventing hypothermia in preterm infants: a randomized trial. J Pediatr. 2014;165(2):261-266.e1.

24. Oatley HK, Blencowe H, Lawn JE. The effect of coverings, including plastic bags and wraps, on mortality and morbidity in preterm and fullterm neonates. J Perinatol. 2016;36(Suppl 1):S83-S89.

25. Carroll PD, Nankervis CA, Giannone PJ, et al. Use of polyethylene bags in extremely low birth weight infant resuscitation for the prevention of hypothermia. J Reprod Med. 2010;55(1-2):9-13.

26. Billimoria Z, Chawla S, Bajaj M, et al. Improving admission temperature in extremely low birth weight infants: a hospital-based multi-intervention quality improvement project. J Perinat Med. 2013;41(4):455-460.

27. Smith J, Usher K, Alcock G, et al. Application of plastic wrap to improve temperatures in infants born less than 30 weeks gestation: a randomized controlled trial. Neonatal Netw. 2013;32(4):235-245.

28. Belsches TC, Tilly AE, Miller TR, et al. Randomized trial of plastic bags to prevent term neonatal hypothermia in a resource-poor setting. Pediatrics. 2013;132(3):e656-e661.

29. Pinheiro JMB, Furdon SA, Boynton S, et al. Decreasing hypothermia during delivery room stabilization of preterm neonates. Pediatrics. 2014;133(1):e218-e226.

30. Bhat SR, Meng NF, Kumar K, et al. Keeping babies warm: a noninferiority trial of a conductive thermal mattress. Arch Dis Child Fetal Neonatal Ed. 2015;100(4):F309-F312.

31. Manani M, Jegatheesan P, DeSandre G, et al. Elimination of admission hypothermia in preterm very low-birth-weight infants by standardization of delivery room management. Perm J. 2013; 17(3):8-13.

32. Srivastava S, Gupta A, Bhatnagar A, et al. Effect of very early skin to skin contact on success at breastfeeding and preventing early hypothermia in neonates. Indian J Public Health. 2014; 58(1):22-26.

33. Nimbalkar SM, Patel VK, Patel DV, et al. Effect of early skin-toskin contact following normal delivery on incidence of hypothermia in neonates more than $1800 \mathrm{~g}$ : randomized control trial. J Perinatol. 2014;34(5):364-368.

34. Moore ER, Anderson GC, Bergman N, et al. Early skin-to-skin contact for mothers and their healthy newborn infants. Cochrane Database Syst Rev. 2012;(5):CD003519.

35. Gouchon S, Gregori D, Picotto A, et al. Skin-to-skin contact after cesarean delivery: an experimental study. Nurs Res. 2010;59(2):78-84.

36. Beiranvand S, Valizadeh F, Hosseinabadi R, et al. The Effects of Skin-toSkin Contact on Temperature and Breastfeeding Successfulness in FullTerm Newborns after Cesarean Delivery. Int J Pediatr .2014:846486.
37. Bergman NJ, Linley LL, Fawcus SR. Randomized controlled trial of skin-to-skin contact from birth versus conventional incubator for physiological stabilization in 1200- to 2199-gram newborns. Acta Paediatr. 2004;93(6):779-785.

38. Knobel RB. Role of effective thermoregulation in premature neonates. Research and Reports in Neonatology. 2014;2014(4):147-156.

39. Te Pas AB, Lopriore E, Dito I, et al. Humidified and heated air during stabilization at birth improves temperature in preterm infants. Pediatrics. 2010;125(6):e1427-e1432

40. Meyer MP, Hou D, Ishrar NN, et al. Initial respiratory support with cold, dry gas versus heated humidified gas and admission temperature of preterm infants. J Pediatr. 2015;166(2):245-250.e1

41. Adamkin DH. Postnatal glucose homeostasis in late-preterm and term infants. Pediatrics. 2011; 127(3):575-579.

42. Zhou W, Yu J, Wu Y, et al. Hypoglycemia incidence and risk factors assessment in hospitalized neonates. J Matern Fetal Neonatal Med. 2015;28(4):422-425

43. Flores-le Roux JA, Sagarra E, Benaiges D, et al. A prospective evaluation of neonatal hypoglycaemia in infants of women with gestational diabetes mellitus. Diabetes Res Clin Pract. 2012;97(2):217-222.

44. García-Patterson A, Aulinas A, María MÁ, et al. Maternal body mass index is a predictor of neonatal hypoglycemia in gestational diabetes mellitus. J Clin Endocrinol Metab. 2012;97(5):1623-1628.

45. Tundidor D, García-Patterson A, María MA, et al. Perinatal maternal and neonatal outcomes in women with gestational diabetes mellitus according to fetal sex. Gend Med. 2012;9(6):411-417.

46. Kerstjens JM, Bocca-Tjeertes IF, de Winter AF, et al. Neonatal morbidities and developmental delay in moderately preterm-born children. Pediatrics. 2012;130(2):265-272.

47. Duvanel CB, Fawer CL, Cotting J, et al. Long-term effects of neonatal hypoglycemia on brain growth and psychomotor development in smallfor-gestational-age preterm infants. J Pediatr. 1999; 134(4):492-498.

48. Kaiser JR, Bai S, Gibson N, et al. Association Between Transient Newborn Hypoglycemia and Fourth-Grade Achievement Test Proficiency: A Population-Based Study. JAMA Pediatr. 2015; 169(10):913-921.

49. Mola-Schenzle E, Staffler A, Klemme M, et al. Clinically stable very low birthweight infants are at risk for recurrent tissue glucose fluctuations even after fully established enteral nutrition. Arch Dis Child Fetal Neonatal Ed. 2015;100(2):126-131.

50. Bennett C, Fagan E, Chaharbakhshi E, et al. Implementing a protocol using glucose gel to treat neonatal hypoglycemia. Nurs Women's Health. 2016;20(1):64-74

51. Rawat M, Chandrasekharan P, Turkovich S, et al. Oral dextrose gel reduces the need for intravenous dextrose therapy in neonatal hypoglycemia. Biomedicine hub. 2016;1(3).

52. Hegarty JE, Harding JE, Gamble GD, et al. Prophylactic Oral Dextrose Gel for Newborn Babies at Risk of Neonatal Hypoglycaemia: A Randomised Controlled Dose-Finding Trial (the Pre-hPOD Study). PLoS Med. 2016;13(10):1002155.

53. Kutamba E, Lubega S, Mugalu J, et al. Dextrose boluses versus burette dextrose infusions in prevention of hypoglycemia among preterms admitted at Mulago Hospital: an open label randomized clinical trial. Afr Health Sci. 2012;14(3):502-509.

54. Ley D, Romantsik O, Vallius S, et al. High presence of extracellular hemoglobin in the periventricular white matter following preterm intraventricular hemorrhage. Front Physiol. 2016;7:330.

55. Demirbilek H, Rahman SA, Buyukyilmaz GG, et al. Diagnosis and treatment of hyperinsulinaemic hypoglycaemia and its implications for paediatric endocrinology. Int J Pediatr Endocrinol. 2017; 9. 
56. McCrea HJ, Ment LR. The diagnosis, management, and postnatal prevention of intraventricular hemorrhage in the preterm neonate. Clin Perinatol. 2008;35(4):777-792.

57. Patra A, Bhandary P, Giannone P. The Golden hour : Providing Very Premature Infants a Favourable Beginning. J Pediatr Neonatal Care. 2016;5(3):00182.

58. Kalani M, Shariat M, Khalesi N, et al. A comparison of early ibuprofen and indomethacin administration to prevent intraventricular hemorrhage among preterm infants. Acta Med Iran. 2016;54(12):788-792.

59. Bruschettini M, Romantsik O, Zappettini S, et al. Antithrombin for the prevention of intraventricular hemorrhage in very preterm infants. Cochrane Database Syst Rev. 2016;3: CD011636.

60. Bruschettini M, Romantsik O, Zappettini S, et al. Heparin for the prevention of intraventricular haemorrhage in preterm infants. Cochrane Database Syst Rev. 2016;5(5):CD011718.

61. Reuter S, Moser C, Baack M. Respiratory distress in the newborn. Pediatr Rev. 2014;35(10):417-428.

62. Rojas-Reyes MX, Morley CJ, Soll R. Prophylactic versus selective use of surfactant in preventing morbidity and mortality in preterm infants. Cochrane Database Syst Rev. 2012;(3):CD000510.

63. Perlman JM, Wyllie J, Kattwinkel J, et al. Part 11: Neonatal resuscitation: 2010 International Consensus on Cardiopulmonary Resuscitation and Emergency Cardiovascular Care Science With Treatment Recommendations. Circulation. 2010;122(16 Suppl 2):516-538.

64. Patel PN, Banerjee J, Godambe SV. Resuscitation of extremely preterm infants - controversies and current evidence. World journal of clinical pediatrics. 2016;5(2):151-158.

65. Preterm Birth: Causes, Consequences, and Prevention. Committee on Understanding Premature Birth and Assuring Healthy Outcomes Board on Health Sciences Policy Richard E Behrman \& Adrienne Stith Butler (Eds.), The National Academic Press, Washington. 2007.

66. Schmölzer GM, Kumar M, Aziz K, et al. Sustained inflation versus positive pressure ventilation at birth: a systematic review and metaanalysis. Arch Dis Child Fetal Neonatal Ed. 2015;100:361-F368.

67. Oei JL, Vento M, Rabi Y, et al. Higher or lower oxygen for delivery room resuscitation of preterm infants below 28 completed weeks gestation: a meta-analysis. Arch Dis Child Fetal Neonatal Ed. 2017;102(1):24-30.

68. Chang M. Optimal oxygen saturation in premature infants. Korean $J$ Pediatr. 2011;54(9):359-362.

69. Oei JL, Saugstad OD, Lui K, et al. Targeted oxygen in the resuscitation of preterm infants, a randomized clinical trial. Pediatrics. 2017;139(1).
70. Wei QZ, Su P, Han JT, et al. [Effect of early caffeine treatment on the need for respirator therapy in preterm infants with respiratory distress syndrome]. Zhongguo Dang Dai Er Ke Za Zhi. 2016; 18(12):1227-1231.

71. Cayabyab R, Arora V, Wertheimer F, et al. Graded oxygen saturation targets and retinopathy of prematurity in extremely preterm infants. Pediatr Res. 2016;80(3):401-406.

72. Blencowe H, Lawn JE, Vazquez T, et al. Preterm-associated visual impairment and estimates of retinopathy of prematurity at regional and global levels for 2010. Pediatr Res. 2013;1:35-49.

73. Beharry KD, Valencia GB, Lazzaro DR, et al. Pharmacologic interventions for the prevention and treatment of retinopathy of prematurity. Semin Perinatol. 2016;40(3):189-202.

74. Cayabyab R, Ramanathan R. Retinopathy of prematurity: therapeutic strategies based on pathophysiology. Neonatology. 2016;109(4):369-376.

75. Lok JYC, Yip WWK, Luk ASW, et al. Visual outcome and refractive status in first 3 years of age in preterm infants suffered from laser-treated Type 1 retinopathy of prematurity (ROP): a 6-year retrospective review in a tertiary centre in Hong Kong. Int Ophthalmol. 2017.

76. Fang JL, Sorita A, Carey WA, et al. Interventions To Prevent Retinopathy of Prematurity: A Meta-analysis. Pediatrics. 2016;137(4):1-17.

77. Visser L, Singh R, Young M, et al.Guideline for the prevention, screening and treatment of retinopathy of prematurity (ROP). S Afr Med J. 2012;103(2):116-125.

78. Fierson WM. Screening examination of premature infants for retinopathy of prematurity. American Academy of Pediatrics Section on Ophthalmology, American Academy of Ophthalmology, American Association for Pediatric Ophthalmology and Strabismus, American Association of Certified Orthoptists. Pediatrics. 2013;131(1):189-195.

79. Kennedy KA, Wrage LA, Higgins RD, et al. Evaluating retinopathy of prematurity screening guidelines for 24- to 27-week gestational age infants. J Perinatol. 2014;34(4):311-318.

80. Langdon A, Crook N, Dantas G. The effects of antibiotics on the microbiome throughout development and alternative approaches for therapeutic modulation. Genome Med. 2016;8(1):39.

81. Chitty H, Wyllie J. Importance of maintaining the newly born temperature in the normal range from delivery to admission. Semin Fetal Neonatal Med. 2013;18(6):362-368.

82. Flenady VJ, Woodgate PG. Radiant warmers versus incubators for regulating body temperature in newborn infants. Cochrane Database Syst Rev. 2002;(2):CD000435.

83. Weiner GM, Zaichkin J, Kattwinkel J. Textbook of Neonatal Resuscitation (7th edn), Elk Grove Village, IL: American Academy of Pediatrics and American Heart Association, USA. 2016. 